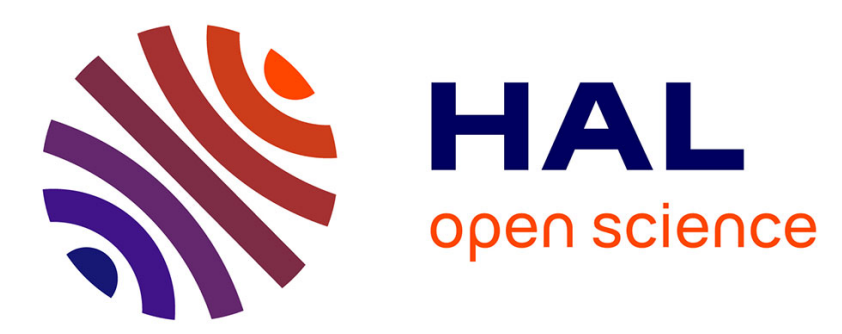

\title{
Detection of ozone in a $\mathrm{MHz}$ argon plasma bullet jet
}

S. Reuter, Joern Winter, S. Iseni, S. Peters, A. Schmidt-Bleker, M. Dünnbier, J. Schäfer, R. Foest, K.-D. Weltmann

\section{To cite this version:}

S. Reuter, Joern Winter, S. Iseni, S. Peters, A. Schmidt-Bleker, et al.. Detection of ozone in a $\mathrm{MHz}$ argon plasma bullet jet. Plasma Sources Science and Technology, 2012, 21 (3), 10.1088/09630252/21/3/034015. hal-02270228

\section{HAL Id: hal-02270228 \\ https://hal.science/hal-02270228}

Submitted on 26 Jun 2021

HAL is a multi-disciplinary open access archive for the deposit and dissemination of scientific research documents, whether they are published or not. The documents may come from teaching and research institutions in France or abroad, or from public or private research centers.
L'archive ouverte pluridisciplinaire HAL, est destinée au dépôt et à la diffusion de documents scientifiques de niveau recherche, publiés ou non, émanant des établissements d'enseignement et de recherche français ou étrangers, des laboratoires publics ou privés. 


\title{
Detection of ozone in a MHz argon plasma bullet jet
}

\author{
S Reuter ${ }^{1,2}$, J Winter ${ }^{1,2}$, S Iseni ${ }^{1,2}, \mathbf{S}$ Peters $^{2}$, A Schmidt-Bleker ${ }^{1,2}$, \\ M Dünnbier ${ }^{1,2}$, J Schäfer ${ }^{2}$, R Foest $^{2}$ and K-D Weltmann ${ }^{2}$ \\ ${ }^{1}$ Centre for Innovation Competence plasmatis, 17489 Greifswald, Germany \\ ${ }^{2}$ Leibniz Institute for Plasma Science and Technology (INP), 17489 Greifswald, Germany \\ E-mail: Stephan.Reuter@inp-greifswald.de
}

Received 28 October 2011, in final form 30 March 2012

Published 16 May 2012

\begin{abstract}
This study for the first time confirms the presence of plasma bullets in a $\mathrm{MHz}$ argon atmospheric pressure plasma jet. Bullet characteristics are investigated by phase-resolved optical emission measurements. Regarding the jet's reactive component output, its ozone production rates are investigated by two independent diagnostic techniques yielding complementary results. The first method-UV-absorption spectroscopy in the Hartley band-determines space-resolved distribution of the ozone concentration in the jet effluent. The second method-quantum cascade laser-absorption spectroscopy in the mid-infrared spectral region-yields high sensitivity results of the average ozone concentration in a multipass cell, in which the effluent is directed. The results of both diagnostic techniques show excellent agreement.
\end{abstract}

\section{Introduction}

Due to progress in recent years in the development and research of non-equilibrium atmospheric pressure plasmas with high reactive species densities at low gas temperature, plasma treatment of sensitive surfaces has become possible. Most importantly, the possibility of treating living tissue promises frontier-breaking therapeutic approaches in the new field of plasma medicine [1-3]. For a thorough analysis of the interaction of cold plasmas with sensitive biological materials, a detailed quantitative analysis of the discharges dynamics as well as the reactive component output is required. Excellently suited for this analysis are non-invasive diagnostic techniques $[4,5]$.

In this work a MHz argon atmospheric pressure plasma jet is investigated. Phase-resolved optical emission spectroscopy yields insight into its excitation dynamics and it can be shown for the first time that even at $\mathrm{MHz}$ operating frequency, plasma bullets are responsible for the effluent dynamics. Regarding the reactive species, this study focuses on the ozone output of the jet. The ozone density in the jet effluent is determined by UV-absorption spectroscopy in the Hartley band and by quantum cascade laser-absorption spectroscopy in the mid- infrared (IR) spectral region. UV-absorption spectroscopy can determine space-resolved ozone concentrations, while quantum cascade laser-absorption spectroscopy has a high sensitivity and determines the spatially averaged ozone output of the jet with high accuracy, so that even the ozone generation from ambient air oxygen diffusing into the plasma jet's effluent at zero oxygen admixture to the feed gas can be quantified. From the UV-absorption spectroscopy measurements a twodimensional map of the optical depth of the ozone absorption is recorded, from which a map of the ozone distribution is calculated via Abel transformation.

Previous approaches to operate the plasma in a controlled noble gas atmosphere in order to have a simplified environment for intricate diagnostics such as TALIF spectroscopy significantly change the chemistry in the effluent. In the present investigations, the plasma jet is operated under realistic conditions in ambient air.

\section{Atmospheric pressure argon plasma jet}

A concentric atmospheric pressure argon plasma jet $\left(\mathrm{kINPen09}{ }^{\odot}\right)$ is investigated. In the center of a ceramic capillary (inner radius $r_{0}=0.8 \mathrm{~mm}$ ) a pin-type electrode 


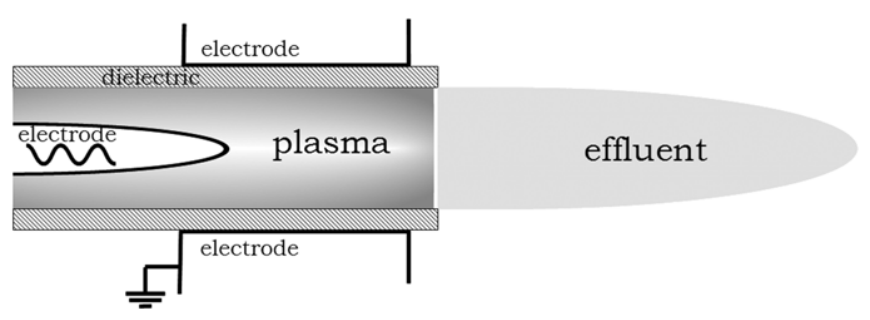

Figure 1. Schematics of the argon plasma jet (kINPen09).

( $0.5 \mathrm{~mm}$ radius) is mounted (see figure 1$)$. In the continuous working mode, a high-frequency sinusoidal voltage $(1 \mathrm{MHz}$, $2-6 \mathrm{kV}_{\mathrm{pp}}$ ) is coupled to the pin-type electrode [6]. The plasma jet is operated with a dry argon flux of 5 standard liters per minute (slm) and in this work with up to $2 \%$ molecular oxygen admixture. Additional technical details about the working conditions and the design of the jet have been presented in previous papers [7].

\section{Phase-resolved optical emission measurements}

Phase-resolved optical emission measurements can image complex plasma dynamics in the ns range and yield valuable information about the plasma chemistry [8]. As non-intrusive plasma diagnostic this technique can also provide access to quenching coefficients and excitation processes [9].

\subsection{Experimental setup}

The experimental setup consists of a high-repetition rate gated iCCD camera (LaVision PicoStar HR12) with $1370 \times 1040$ pixels and a mounted macro-objective, $25 \mathrm{~cm}$ in front of the plasma jet. The trigger of the camera's gate is - via a delay generator-connected to the trigger output of the plasma jet's $1 \mathrm{MHz}$ power supply. Because of the setup's simplicity, a schematic is not shown. The system has a spatial resolution of approximately $30 \mu \mathrm{m}$. For phase-resolved measurements the iCCD camera is triggered synchronously to the excitation signal of the plasma jet. At a fixed phase position of the excitation signal, the light emission from the visible effluent is acquired with a gate width of $1 \mathrm{~ns}$, as shown in figure 2 . The emission from all cycles within a time frame of $700 \mathrm{~ms}$ was integrated.

After this time integration the phase is shifted by a defined time step of $25 \mathrm{~ns}$ with respect to the phase-locked trigger, so that one full cycle with a period of $1 \mu \mathrm{s}$ is resolved into 41 equidistant time intervals.

\section{2. $\mathrm{MHz}$ plasma bullets}

Figure 3 shows a side view of the propagating argon effluent as spatial intensity distribution at a specific time of the excitation cycle. The tip of the outer ceramic capillary is illuminated by the discharge and the horizontal white dotted line indicates the position where the emission of the effluent is analyzed phase resolved. Accordingly, all deduced axial intensity profiles of the emission are arranged as color-coded 2D plots over time, shown in figure 4 . These non-interpolated raw data reveal the

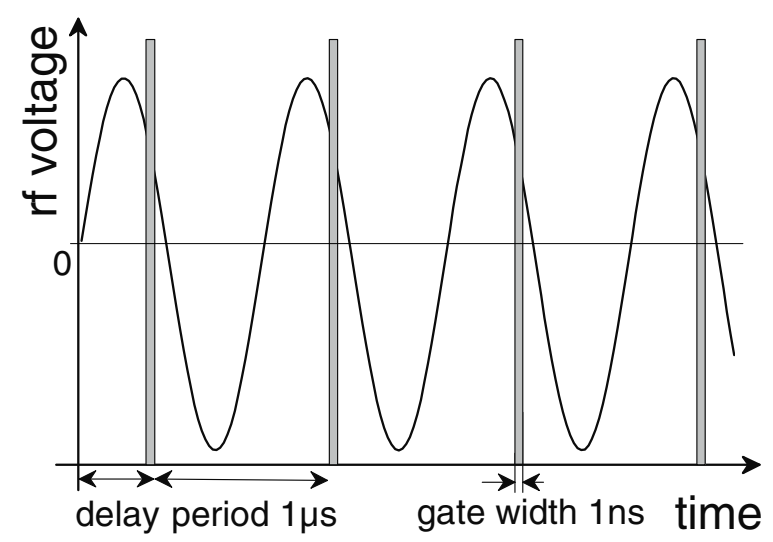

Figure 2. Principle of phase-resolved optical emission spectroscopy.

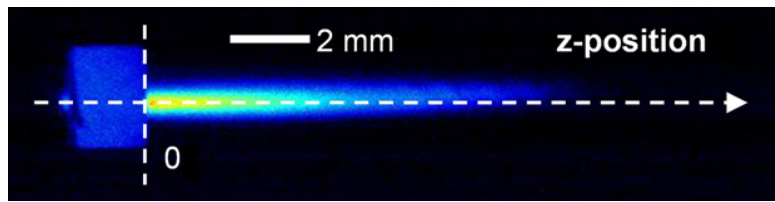

Figure 3. Space-resolved wavelength integrated emission profile. The image is false color-coded. Zero denotes the tip of the nozzle. The jet is operated with $5 \mathrm{slm}$ argon and no oxygen admixture. Integration time is $700 \mathrm{~ms}$.

discharge dynamics. The transition from the illuminated tip of the outer ceramic capillary ( $z$-positions below $0 \mathrm{~mm}$ ) and the free effluent can clearly be observed in all contour plots as a stepwise increase of intensity. The remarkable characteristic, however, is the discontinuous effluent, indicating the presence of so-called plasma bullets, known in the literature for the case of $\mathrm{kHz}$ excitation [10-12]. These bullets become more pronounced for higher oxygen admixtures. The emission intensities in the first and second half-wave of the excitation cycle decrease dramatically to zero with increasing oxygen admixture in the case of the first half-wave.

For pure argon the plasma bullet evolves between 0.4 and $0.6 \mu \mathrm{s}$ and detaches from the effluent. The black dashed line in figure 4 indicates the spatio-temporal development of the bullet and the lines slope represents the bullet velocity. The temporal position of the bullet $t_{\mathrm{b}}(z)=\int_{t_{0}}^{t_{1}} \mathrm{~d} t t I_{z}(t)$ with $t_{0}=T / 4$ and $t_{1}=\frac{3}{4} \mathrm{~T}$ is calculated for $z \leqslant 4 \mathrm{~mm}$. $I_{z}(t)$ is the emission intensity normalized to unity. This yields the bullet trajectory $z\left(t_{\mathrm{b}}\right)$ from which the bullet velocity can be derived via linear regression. Bullet velocities for different oxygen admixtures are shown in figure 5.

In pure argon the bullet velocity reaches up to $7 \times$ $10^{4} \mathrm{~m} \mathrm{~s}^{-1}$. Admixing only 5 standard cubic centimeter $(\mathrm{sccm})$ oxygen, the bullet velocity is almost reduced to half of the initial value. With an oxygen admixture of $20 \mathrm{sccm}$ the bullet velocity is reduced by about $80 \%$. Toward higher oxygen admixtures, the bullet velocity converges to a level of approximately $1.1 \times 10^{4} \mathrm{~m} \mathrm{~s}^{-1}$. These results agree with reported bullet velocities ranging from $10^{3}$ up to $10^{5} \mathrm{~m} \mathrm{~s}^{-1}$ for 'bullet-like' structures in other discharges [13-17]. Moreover, the velocity is not constant for the case of no oxygen admixture and for $5 \mathrm{sccm} \mathrm{O}_{2}$ admixture at the end of the effluent, in contrast to the other slopes for higher oxygen admixtures. 

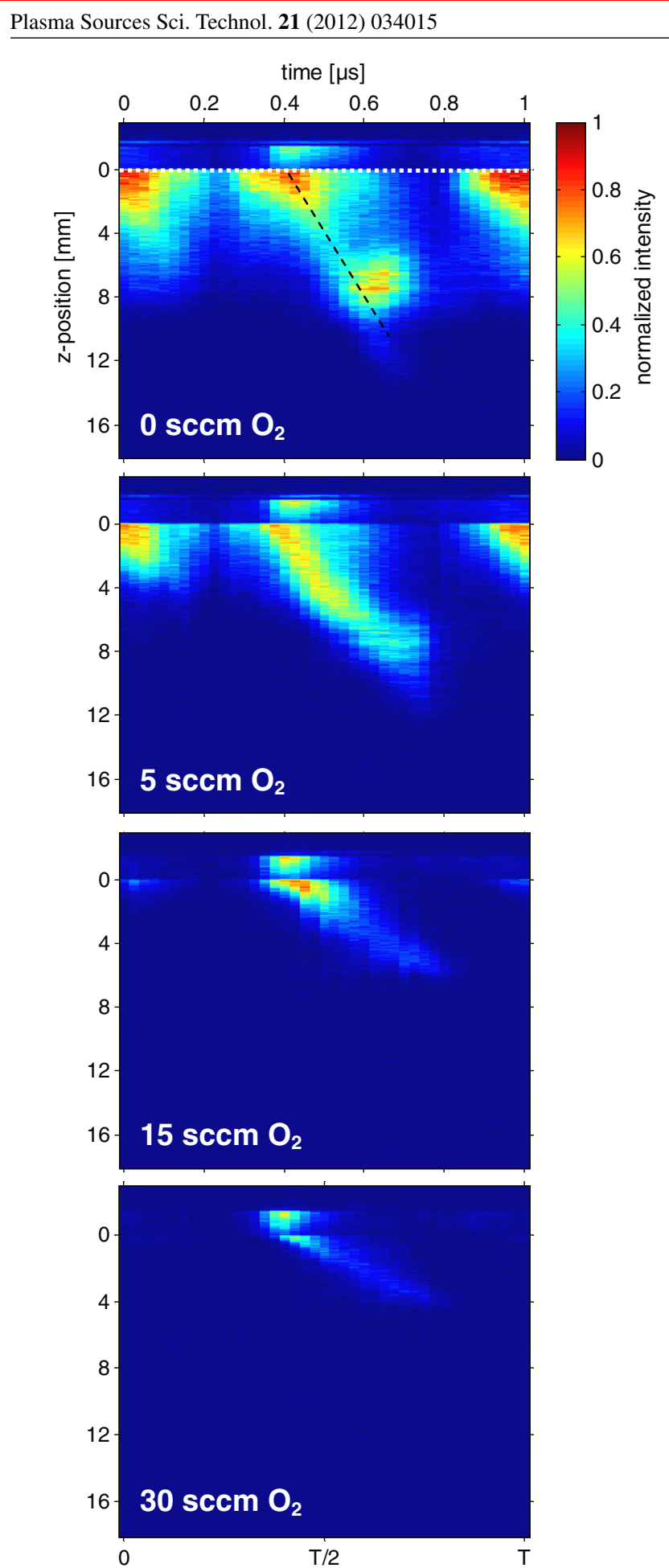

Figure 4. Phase- and space-resolved wavelength integrated emission profiles in the visible spectral range for different oxygen admixtures over one excitation period $\mathrm{T}$.

This observed non-linearity can be due to a factual change of the bullet velocity or it can be an overlying feature of a front bullet and an occurring back bullet, as seen in a $\mathrm{kHz}$ driven plasma needle [18]. This back bullet can assumedly be seen in the round-shaped structure at $8 \mathrm{~mm} z$-position in the contour plot without oxygen admixture. For an elucidation of back bullets in the $\mathrm{MHz}$ plasma jet, future investigations with a higher temporal resolution will be performed.

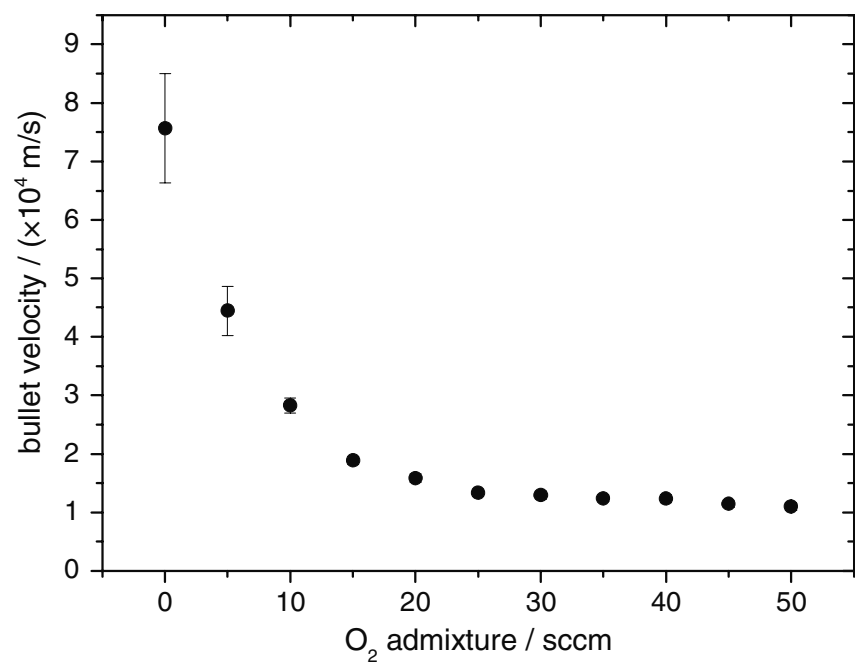

Figure 5. Plasma bullet velocity dependent on $\mathrm{O}_{2}$ admixture.

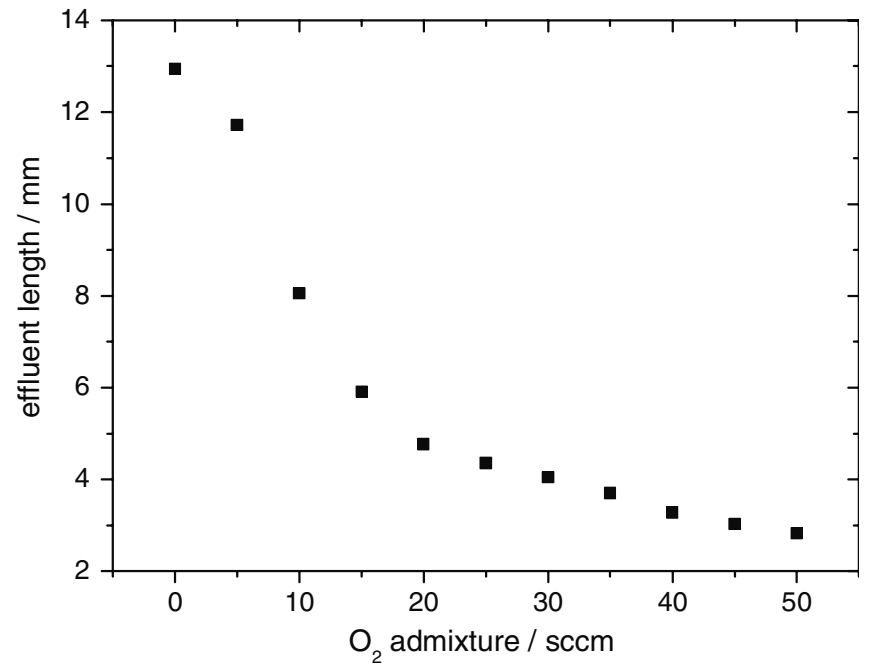

Figure 6. Variation in effluent length dependent on $\mathrm{O}_{2}$ admixture.

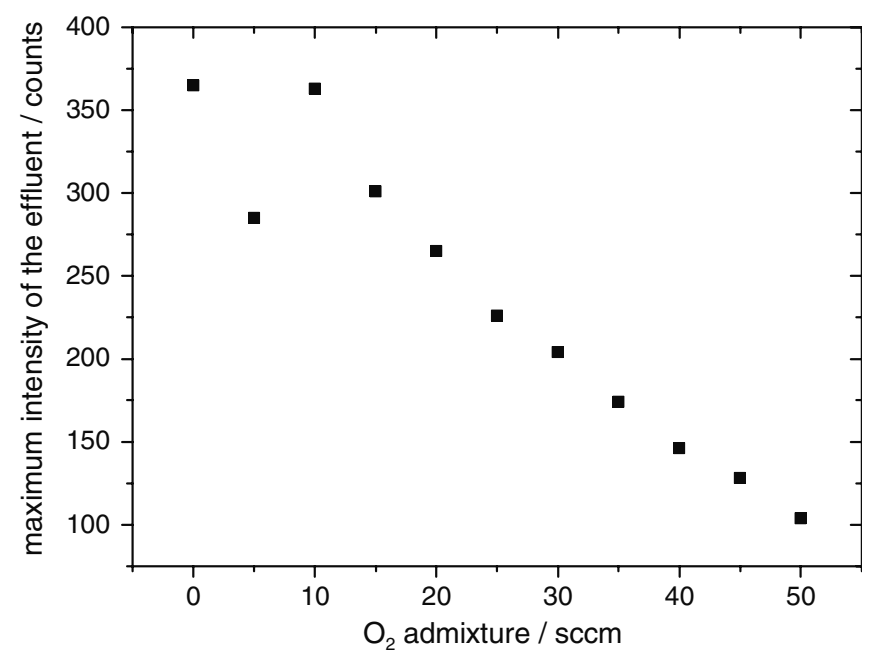

Figure 7. Dependence of the maximum emission intensity of the effluent on $\mathrm{O}_{2}$ admixture. 


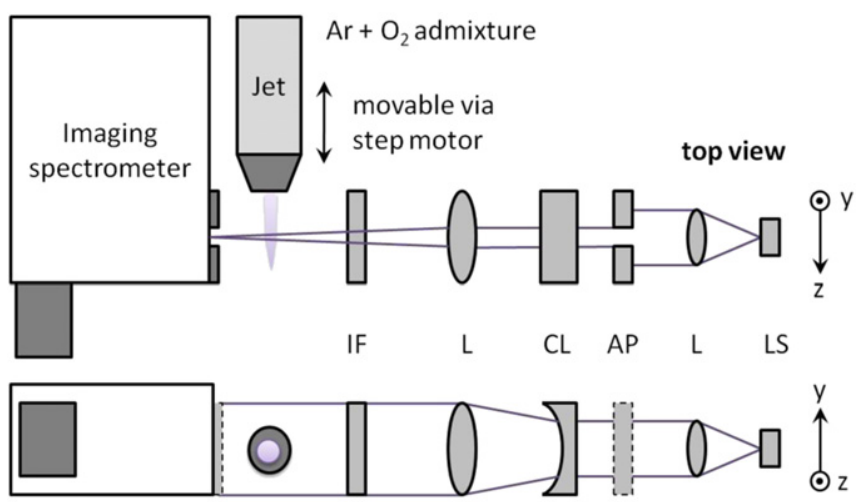

side view

Figure 8. Experimental setup for the spatial-resolved ozone concentration measurement. LS: UV-light source, L: lens, AP: aperture, CL: cylinder lens, IF: interference filter.

As shown in figures 6 and 7, both the length and the maximum intensity of the effluent emission drop with increasing oxygen admixture.

\section{Ozone detection by UV-absorption spectroscopy}

\subsection{Experimental setup}

The spatially resolved absorption signal of ozone in the jet effluent is determined by the UV-absorption setup presented in figure 8. Here, the commonly used absorption of ozone at the wavelength of $253.7 \mathrm{~nm}$ is taken as a measure for the ozone concentration. A deuterium lamp (DH-2000, Ocean Optics) is used as background source. This lamp emits a broadband spectrum of UV light in the range from 200 to $300 \mathrm{~nm}$. To ensure that only photons with a wavelength of around $254 \mathrm{~nm}$ are applied for the absorption measurements an interference filter with a central transmission wavelength of $254 \mathrm{~nm}$ and full width at half maximum (FWHM) of $10 \mathrm{~nm}$ is inserted into the setup between the lamp and the plasma jet. A UV beam sheath is generated by means of an aperture, biconvex lenses, and a cylinder lens and focused onto the entrance slit $(400 \mu \mathrm{m})$ of a $0.5 \mathrm{~m}$ imaging spectrometer (Shamrock 500i, grating: 2400 lines $\mathrm{mm}^{-1}$, blazed at $300 \mathrm{~nm}$, Andor). An iCCD camera (iStar, Andor) is connected to the spectrometer as a detector. This setting provides a sensitive and spectrally resolved detection of UV light around $254 \mathrm{~nm}$. The exposure time of the camera is $100 \mathrm{~ms}$.

The plasma jet is positioned orthogonally to the UV beam sheath on a two-axis stepping motor system. Hence, the jet can be precisely moved in the axial and vertical directions. The spatial resolution in the axial $(z)$ and vertical directions $(y)$ is determined by the sheath-thickness and the resolution of the iCCD camera and amounts to $0.5 \mathrm{~mm}$ and $0.2 \mathrm{~mm}$, respectively. Using an imaging spectrometer, the spatially resolved absorption signal in the vertical direction (along the spectrometer's entrance slit) can be detected instantaneously while the axial distribution has to be determined by moving the $z$-axis motor stepwise. The axial step size is set to $2 \mathrm{~mm}$. The starting point $(z=0 \mathrm{~mm})$ is the tip of the jet nozzle and the largest distance from the nozzle was $20 \mathrm{~mm}$. The $y$-axis stepping motor is only used for a radial adjustment of the jet.

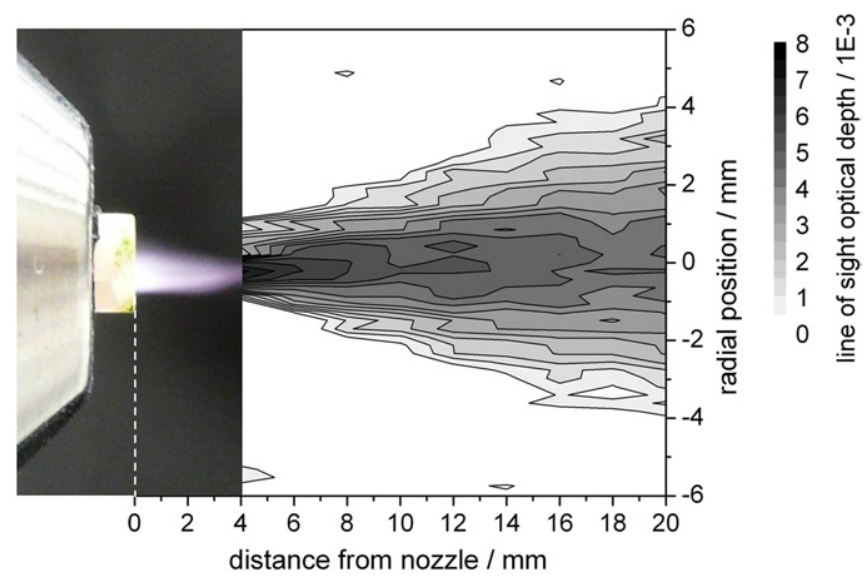

Figure 9. Spatial line-of-sight optical depth of ozone in the vicinity of the jet effluent resulting from the UV-absorption measurement. Due to plasma-induced UV beam distraction no reliable data are obtained for nozzle distances below $4 \mathrm{~mm}$. On the left-hand side of the figure a scaled photograph of the jet and the effluent is shown.

Prior to the absorption measurement, the plasma jet is driven for $20 \mathrm{~min}$ with a gas flow mixture of $5 \mathrm{slm}$ of pure argon (99.999\%) and a small admixture of $25 \mathrm{sccm}$ of pure oxygen $(99.999 \%)$ to provide stable conditions. For the same reason the UV lamp is switched on several hours before the measurement. To avoid lamp switching during the measurement the internal lamp shutter is used to interrupt the UV beam.

\subsection{Spatial distribution of ozone}

In order to obtain a reliable optical depth profile generally four separate measurements are required. First, the background light emission $I_{\mathrm{b}}$ has to be determined. This is carried out by measuring the stray light intensity with the jet and the UV lamp switched off. In the second measurement the emission $I_{\mathrm{p}}$ of the jet effluent is determined without UV-lamp emission. In the third measurement the absorption intensity $I$ is detected, where both, the plasma and the UV lamp are switched on. In the final measurement, the intensity of the UV lamp is determined without the plasma jet. In order to reduce measurement error an average of 5000 acquisitions were performed for each of the four intensity measurement steps.

From the four measurements the line-of-sight optical depth profile $\tau(y, z)$ can be calculated according to

$$
\tau(y, z)=-\ln \left(\frac{I(y, z)-I_{\mathrm{p}}(y, z)-I_{\mathrm{b}}}{I_{0}(y, z)-I_{\mathrm{b}}}\right) .
$$

This optical depth calculation results in the map displayed in figure 9. Additionally, a photograph of the jet with its effluent is included in the figure to indicate the geometrical relations. Due to the expanding gas flow in the radial direction and the diffusion of ozone from the center of the effluent in the radial direction a funnel-shaped optical depth profile forms. In the axial direction the highest line-of-sight optical depth was detected at the initial measurement position $z=4 \mathrm{~mm}$ at a distance of $4 \mathrm{~mm}$ from the jet nozzle. Its value on the axis at $y=0 \mathrm{~mm}$ amounts $8 \times 10^{-3}$. For increasing distances $z$ 


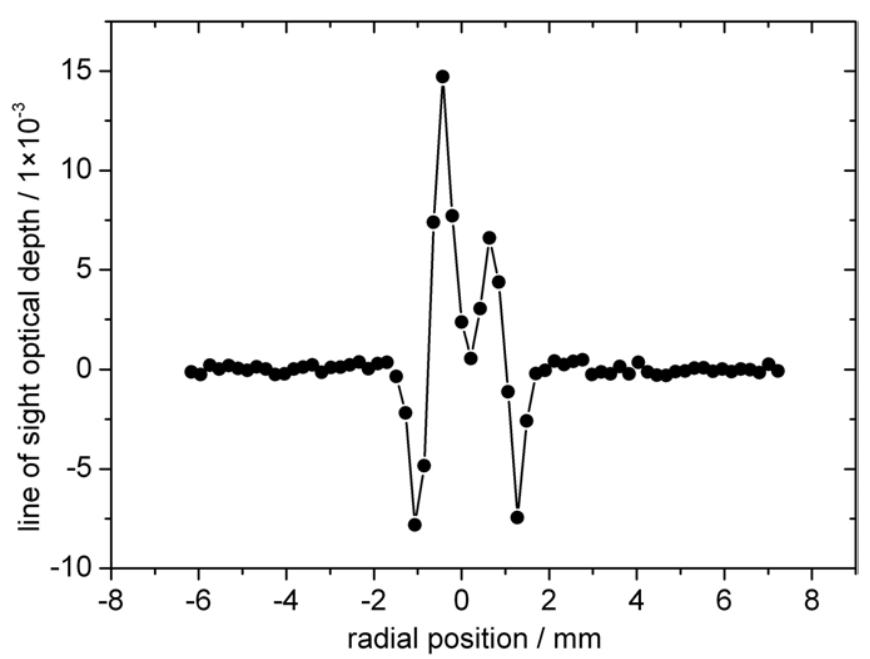

Figure 10. Radial profile of the line-of-sight optical depth measured at a nozzle distance of $z=2 \mathrm{~mm}$.

the optical depth decreases. At a distance of $z=20 \mathrm{~mm}$ the optical depth value on the axis drops to $4.5 \times 10^{-3}$.

The absorption measurement directly inside the visible effluent region (below $z=4 \mathrm{~mm}$ ) turns out to be difficult since plasma-induced temperature variations lead to a refractive index change and in consequence to a distraction of the UV beam. In fact, the cylindrical visible effluent shape acts as a convex lens that strongly refracts UV light from the center to the outer regions. This diagnostic problem is emphasized in figure 10, where the radial-dependent line-of-sight optical depth $\tau(y)$ measurement is shown for a nozzle distance of $z=2 \mathrm{~mm}$. In the outer radial regions negative optical depth values are detected. This means that the absorption intensity measured at these radial positions must be higher than the background intensity emitted from the deuterium lamp. Furthermore, around $r=0 \mathrm{~mm}$ an additional dip is obtained. Both observations are in agreement with the above-discussed lens effect. However, the correction of this refracting effect is sophisticated and intended for further investigations. In this paper, the data obtained for nozzle distances below $z=4 \mathrm{~mm}$ are not used for evaluation.

Outside the visible effluent region $(z \geqslant 4 \mathrm{~mm})$ reasonable optical depth profiles were measured. Two exemplary profiles are shown in figure 11 for nozzle distances of $z=8 \mathrm{~mm}$ and $z=20 \mathrm{~mm}$. As already discussed for the spatial optical depth map (figure 9) the profile obtained at a smaller nozzle distance is narrower and has a higher amplitude than the profile obtained at $z=20 \mathrm{~mm}$.

The relation between the optical depth $\tau$ and the ozone concentration is given by Beer's law

$$
\tau(z)=\sigma \int_{0}^{L}\left[\mathrm{O}_{3}\right](r, z) \mathrm{d} r,
$$

where $L$ is the total absorption length, $\left[\mathrm{O}_{3}\right](r, z)$ is the ozone concentration in molecules per cubic centimeter and $\sigma=1.14 \times 10^{-17} \mathrm{~cm}^{2}$ is the ozone absorption cross section at a wavelength of $253.7 \mathrm{~nm}$ [19]. Under the assumption of an axisymmetric absorption profile, which is reasonably fulfilled

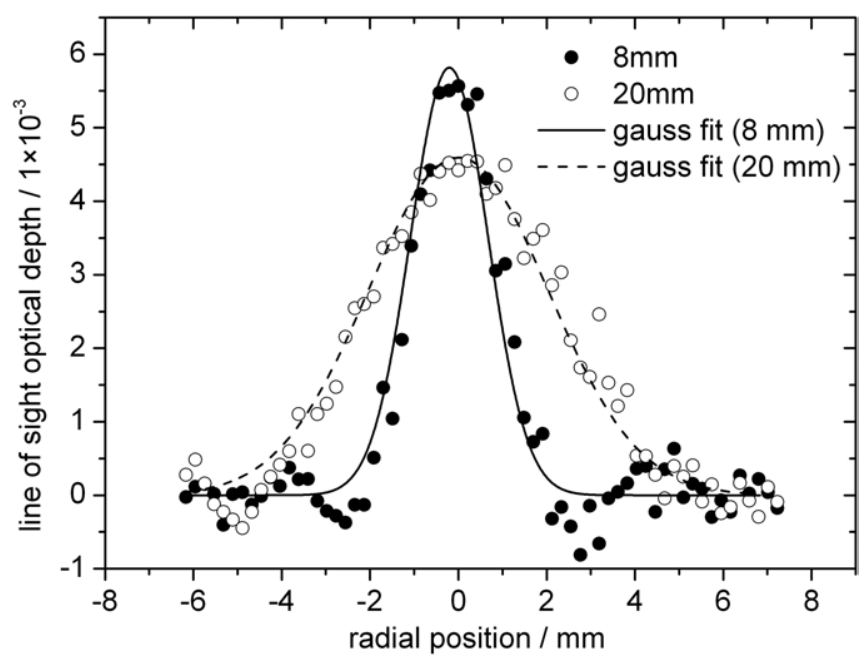

Figure 11. Radial profile of the line-of-sight optical depth measured at a nozzle distance of $z=8 \mathrm{~mm}$ and $z=20 \mathrm{~mm}$. Both profiles were fitted with a Gaussian function.

for the presented measurement, the radial and axial ozone concentration $\left[\mathrm{O}_{3}\right](r, z)$ is calculated numerically using Abel transformation

$$
\left[\mathrm{O}_{3}\right](r, z)=-\frac{1}{\pi \cdot \sigma} \int_{r}^{R} \frac{\mathrm{d} \tau(y, z)}{\mathrm{d} y} \frac{\mathrm{d} y}{\sqrt{y^{2}-r^{2}}},
$$

where $r$ is the coordinate in the radial direction and $R$ is the distance from the axis at that point where the ozone concentration declines to zero (set to $R=7 \mathrm{~mm}$ in the calculation).

Since numerical Abel transformation is sensitive to signal noise, the measured profiles were approximated by Gaussian profile functions as is shown in figure 11. Subsequently these fitted Gaussian functions are Abel transformed instead of the originally measured profiles. The Abel transformation result is displayed in figure 12 . The ozone concentration $\left[\mathrm{O}_{3}\right](r, z)$ is maximal on the axis and declines in the radial direction. Similar to the line-of-sight optical depth profile $\tau(y, z)$ the concentration profile has a funnel shape.

Considering the axial dependence (figure 13) a maximum concentration value of $6.5 \times 10^{15} \mathrm{~cm}^{-3}$ was found at $z=4 \mathrm{~mm}$. From this the concentration declines with increasing nozzle distances to a value of approximately $1 \times 10^{15} \mathrm{~cm}^{3}$ at $z=$ $20 \mathrm{~mm}$.

\section{Detection of ozone by IR-absorption spectroscopy}

\subsection{Experimental setup}

The experimental setup for the ozone concentration measurements by absorption spectroscopy in the middle IR range is presented in figure 14. For these measurements, a commercial absorption spectrometer (Q-MACS, neoplas control [20]) is used, with a specification of the spectral resolution of $0.004 \mathrm{~cm}^{-1}$, which offers a higher resolution than a more commonly used Fourier-transformed IR spectrometer. The IR source is a $2.5 \mathrm{~mW}$ pulsed quantum cascade laser (Alpes Lasers). The center frequency is tuned through 


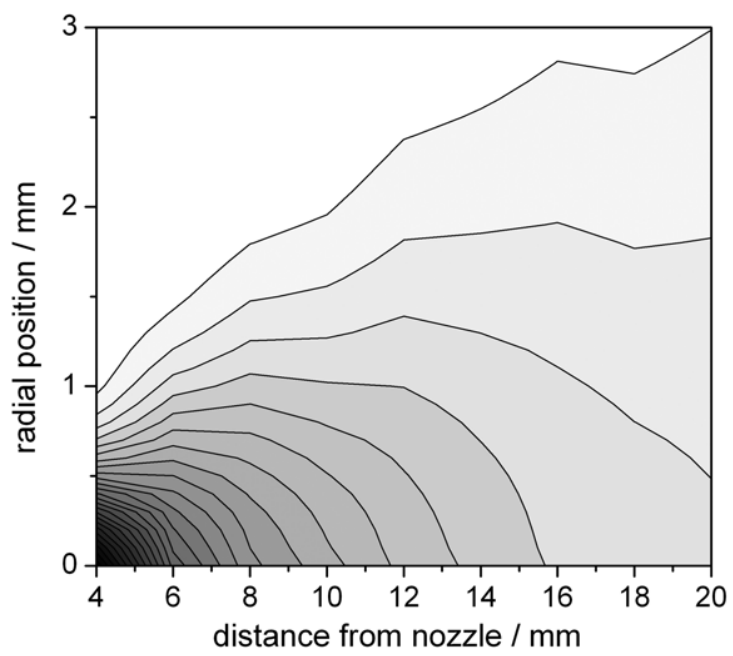

Figure 12. Spatial ozone concentration in the vicinity of the jet nozzle as a result of Abel transformation.

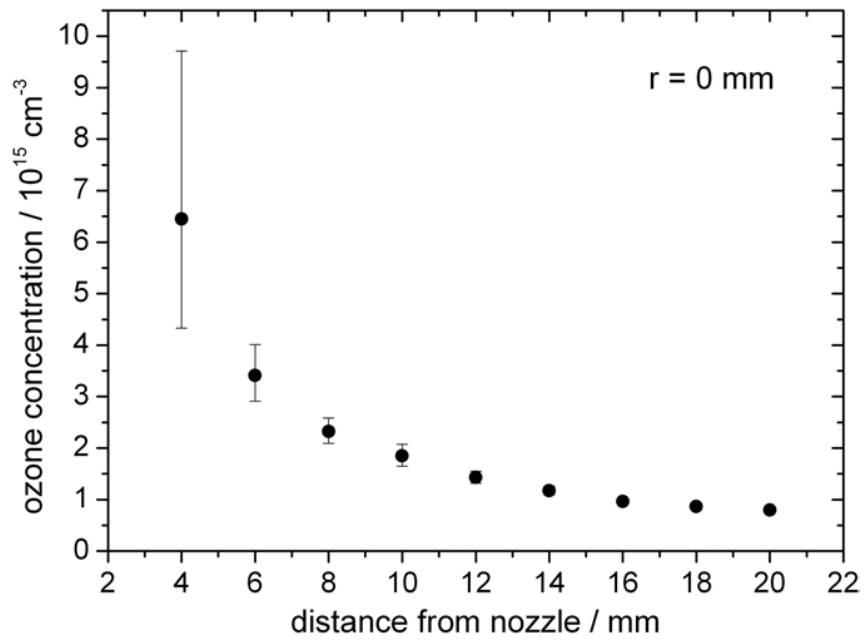

Figure 13. Axial ozone concentration distribution at $r=0 \mathrm{~mm}$.

temperature variation controlled by a Peltier element. The full accessible range is from 1024.5 to $1029.9 \mathrm{~cm}^{-1}$. The IR beam is collimated with an off-axis parabolic mirror and guided to a $1 \mathrm{~m}$ focal length spherical mirror, which focuses the beam on the entrance of a $60 \mathrm{~cm}$ multipass White cell. Thus the total absorption length in the multipass cell is $16.80 \mathrm{~m}$ for 28 paths of the beam. The exit beam is focused on a detector (Q-MACS IRDM-600A) by a further off-axis parabolic mirror. In order to reduce noise and increase efficiency, the detector is cooled by a Peltier element and an external water-cooling unit. In order to align the IR beam, a $5 \mathrm{~mW}$ red laser diode was used. It is assumed that the red and the IR beams follow the same path according to the two apertures set between the laser and the multipass cell. The number of paths is controlled via an adjustable mirror within the cell roughly aligned through the red laser beam. An accurate control is performed with an oscilloscope connected to the detector to measure the delay between the emitted and the detected IR laser pulse. The number of passes can be adjusted between 2 and 28 paths. The multipass cell is at atmospheric pressure and in ambient air to allow the plasma jet guided into the cell to be operated under

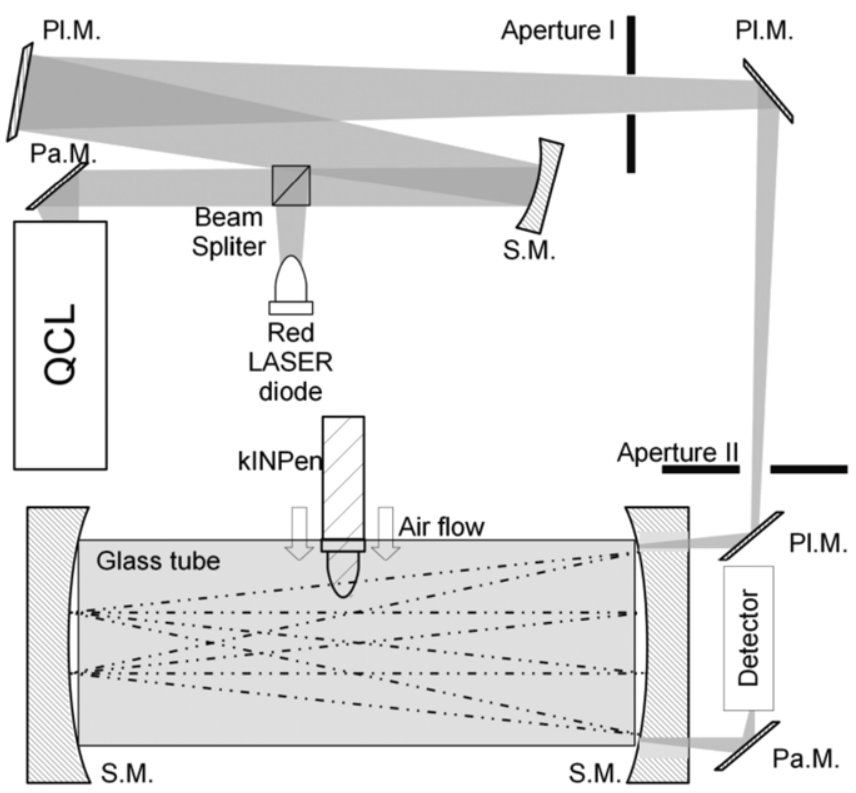

Figure 14. Experimental setup. QCL: quantum cascade laser, Pa.M: parabolic mirror, Pl.M: plan mirror, S.M: spherical mirror.

the same conditions as in open air. A glass tube of $57.5 \mathrm{~cm}$ length is set in the middle of the multipass cell in order to control the atmosphere around the plasma. An inlet of $2 \mathrm{~cm}$ diameter is situated in the middle of the tube in order to insert the jet $2 \mathrm{~cm}$ inside the cell. The sides of the tubes are closed by two plastic disks. Each of these lids has a rectangular cut-out of $15 \mathrm{~cm}^{2}$ as a gas outlet. The argon feed gas flow is fixed at $5 \mathrm{slm}$. In order to avoid saturation of argon within the tube and thus change the ambient conditions, four inlets distributed each $90^{\circ}$ along a $4 \mathrm{~cm}$ diameter circle centered on the plasma jet axis were added. Through these inlets, an additional air gas flow fixed at $10 \mathrm{slm}$ provides a good homogeneity of air surrounding the jet.

The gas was flowing for a considerable time and the plasma jet was switched on at least $1 \mathrm{~h}$ before the first measurement in order to assume dry feed gas and stable conditions. Particular attention was paid to reduce the humidity in the feed gas due to its considerable influence on the jet chemistry. In order to study the influence of oxygen admixture to the feed gas on the production of reactive species, the quantity of 5 to $100 \mathrm{sccm}$ of oxygen $(0.1$ to $2 \%)$ was added to the argon gas flow.

\subsection{Detection of ozone}

It is well known that ozone has strong absorption bands in the IR region [21]. Figure 15 presents the line strength of ozone molecules versus the wavenumber. The probability of absorption is directly proportional to the line strength. Detailed information about the concept of line strength applied here can be found, for example, in [22]. The excitation frequency centered on $1027 \mathrm{~cm}^{-1}$ has been chosen, since the strongest absorption within the spectral range of the measurement setup can be found there.

The laser frequency is calibrated using a reference gas cell filled with a mixture of $95 \%$ argon and 5\% carbonyl 


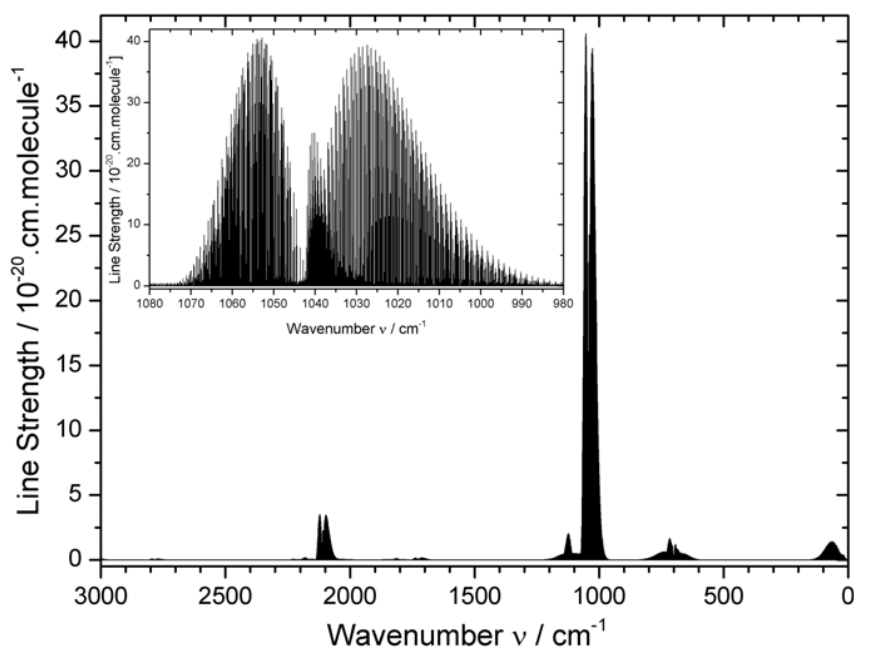

Figure 15. Ozone line strength values retrieved from the HITRAN database [21].

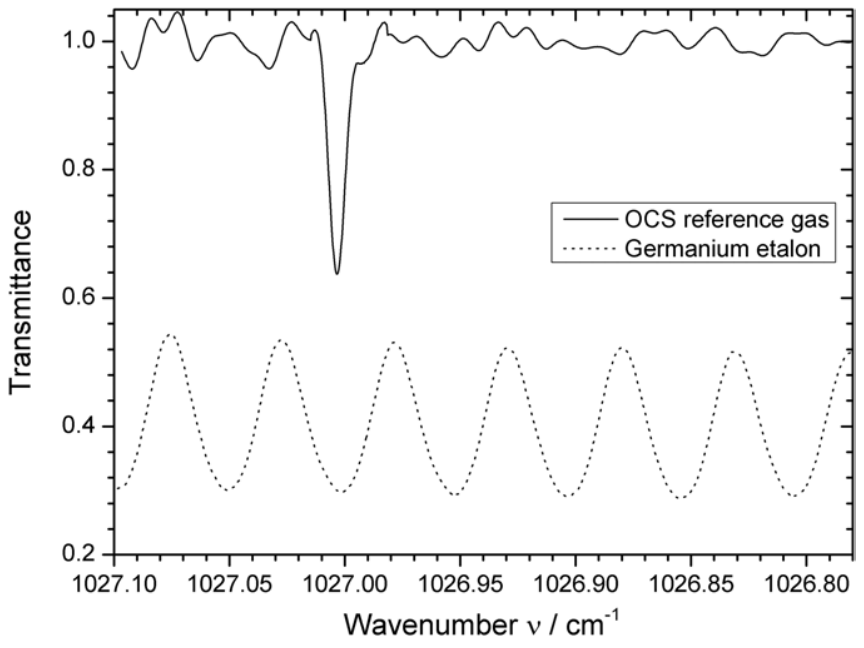

Figure 16. Identification and wavenumber calibration using an OCS gas reference cell together with a spectrum measured with a germanium etalon.

sulfide (OCS). The cell is $15 \mathrm{~cm}$ long and closed by two circular potassium bromide $(\mathrm{KBr})$ windows of $0.5 \mathrm{~cm}$ thickness. The pressure in the cell is set to $10 \mathrm{mbar}$ in order to provide a narrow OCS absorption peak. Figure 16 shows a transmission spectrum of OCS. For this measurement, the gas reference cell is inserted into the multipass cell at 28 paths, which corresponds to $4.2 \mathrm{~m}$ absorption length. It should be noted that the baseline, which corresponds to no absorption, exhibits a wavy structure. It can be assumed that this is due to the $\mathrm{KBr}$ windows, which absorb a part of the IR beam and are also a source of interference. This distortion of the signal does not appear during the ozone measurements, as the IR beam for these measurements passes through the $15 \mathrm{~cm}^{2}$ outlets of the glass tube. The calibration of the wavenumber scale is carried out via a 1 inch germanium $(\mathrm{Ge})$ etalon which acts as a Fabry-Perot interferometer.

Figure 17 shows the transmission spectrum of ozone generated by the plasma jet with a mixture of argon and $2 \%$ of oxygen admixture. On the same graph a simulation of the ozone absorption spectrum is plotted for the same

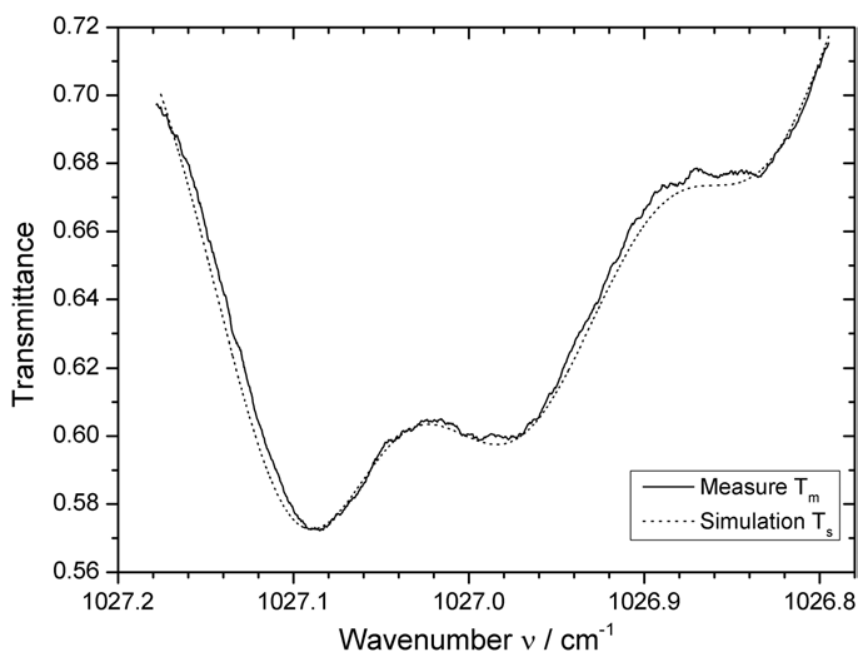

Figure 17. Detection of ozone molecules. Measured spectrum and simulation by Q-MACSoft HT [20] based on HITRAN database [21].

conditions, i.e. $296 \mathrm{~K}, 1013 \mathrm{mbar}$ at $16.8 \mathrm{~m}$ absorption length. The calculations are performed by the commercial available software Q-MACSoft-HT (Neoplas Control [20]). The simulation of the absorption profile is based on the compilation of experimental and theoretical data from the HITRAN database. The algorithm computing the transmittance spectrum $T_{\mathrm{s}}$ is based on Beer-Lambert's law. Equation (4) corresponds to the calculation of the transmittance $T_{1}$ of only one ro-vibrational transition, where $I_{1}(v)$ is the intensity of this single transition, $N$ is the density of the species, $L$ is the absorption length, $S_{1}$ is the transition line strength and $f_{1}\left(v-v_{0}\right)$ is the line shape or function profile of this transition. The implementation of the Doppler broadening-which is very small at atmospheric pressure-as well as the pressure broadening is included in this function profile. According to the concept of line strength, which assumes the Boltzmann distribution, the measure of only one transition enables us to reach the absolute density. However, mainly because of the pressure broadening we cannot measure the transmittance of a single transition. The measured spectrum $T_{\mathrm{m}}$ is actually the sum of many transmittances from all the transitions, which are in the range from 1027.18 to $1026.80 \mathrm{~cm}^{-1}$. In this case, the result of all physical effect is convoluted with the instrumental profile $h\left(v-v_{0}\right)$; see equation (6).

$$
\begin{gathered}
T_{1}(v)=\frac{I_{1}(v)}{I_{0}(v)}=\exp \left[-N L S_{1} f_{1}\left(v-v_{0}\right)\right] \\
T(v)=\sum_{n} T_{n}(v) \\
T_{\mathrm{s}}(v)=T(v) \otimes h(v) .
\end{gathered}
$$

During the acquisition of the spectrum $T_{\mathrm{m}}$, the simulation is running and a good fit to the measurement is achieved by optimization of the concentration value. The good agreement of $T_{\mathrm{m}}$ and $T_{\mathrm{s}}$ shows that the jet in fact produces ozone molecules. This is in contrast to the overview FTIR absorption measurements performed by Pipa et al on a similar, but hotter plasma jet [23] which did not show any sign of ozone. 


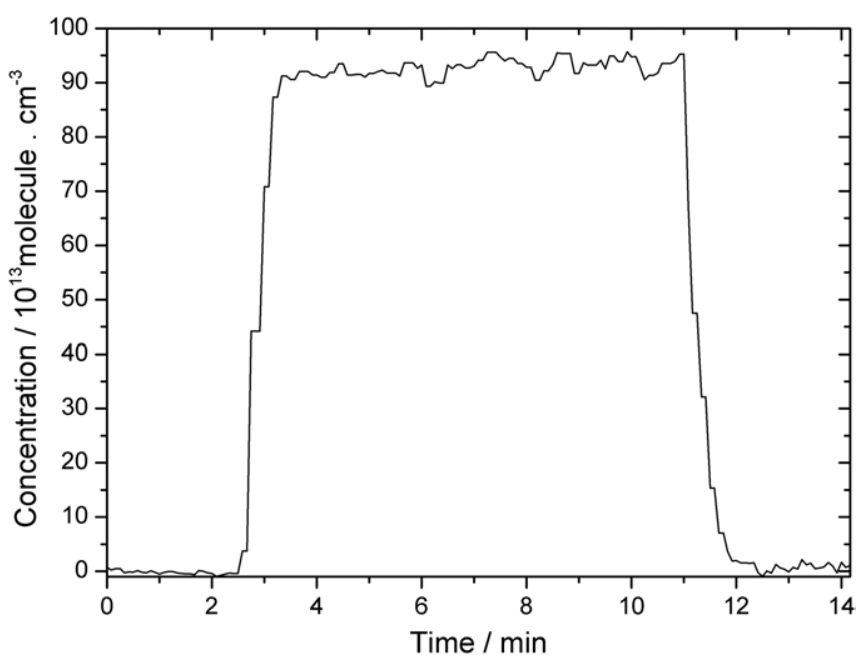

Figure 18. Record of a full measurement procedure. The jet is operated with argon and $2 \%$ of oxygen admixture.

\subsection{Determination of the ozone concentration}

For the measurement of the concentration, the fitting processing is updated every $5 \mathrm{~s}$ and the value of the concentration is recorded. Due to the atmospheric pressure, the broadening of the absorption lines is so significant that the IR radiation is absorbed along the whole observation band. For this reason, the maximal intensity without any absorption is saved in order to be set as baseline for the fitting procedure. The variation of the baseline is less than $0.5 \%$ after $1 \mathrm{~h}$, which ensures a good accuracy of the measurements.

In order to reduce the error of the measurement and to investigate the stability of the jet, the concentration was measured over the time period of a few minutes. The data were statistically processed. Figure 18 shows a complete measurement cycle, which contains three phases: during the 2.5 first minutes the jet is not set into the tube, which provides a background measurement with only the residual ozone from the air. Subsequently, the jet is inserted in the cell, resulting in a significant rise of the ozone concentration. After a few minutes, the jet power supply is switched off but the feed gas is kept running in order to keep the same ratio of argon to air inside the tube. This is also an additional control to check the stability of the laser intensity. The concentration is determined as the average of all the measurements (from 3 to $9 \mathrm{~min}$ ). This average is corrected by the background measurement, which corresponds to the average of the concentration without the jet (from 0 to $2: 30 \mathrm{~min}$ ). The error is then deduced by the sum of the deviations, i.e. the deviation of the background sample and of the measurement sample. For this configuration, the concentration is $(93 \pm 3.5) \times 10^{13} \mathrm{~cm}^{-3}$.

With the above-described setup the influence of oxygen admixture is investigated. Phase-resolved measurements showed that the oxygen admixture causes a reduction of the visible jet length. However, figure 19 shows that the generated ozone concentration increases significantly with increasing oxygen admixture. The production of ozone seems to follow an exponential law and to reach a saturation regime. This will be the topic of a further investigation by a chemical reaction. By the IR-absorption technique we are able to detect and measure

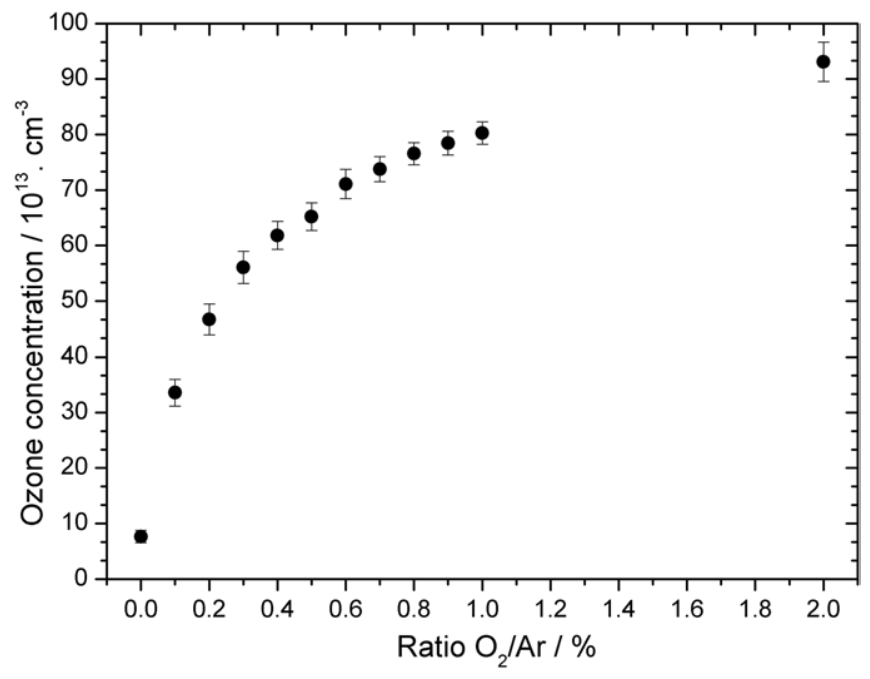

Figure 19. Ozone concentration measured for different ratios of oxygen to argon.

that ozone is also produced with a pure argon gas flow into the jet. We have ensured that there is no humidity in the argon flow, which leads to the fact that the only source of oxygen is the surrounding air. The ozone detected in the cell must therefore be a result of dissociation of indiffusing air oxygen. Further investigations, especially of the diffusion mechanism, should be undertaken in the future.

\section{Discussion}

\subsection{Plasma dynamics and reactive species generation}

The envisaged use of the investigated plasma jet for biomedical applications makes it necessary to consider the question of correct handling and treatment procedure. Biological material is most sensitive to changes under plasma treatment conditions, therefore an adequate distance measure of the plasma jet from the biological sample is frequently discussed in this context. From a practical point of view the visible jet effluent is often used intuitively as a measure of distance and plasma jet efficacy. However, considering the above results of the ozone concentration and the effluent length in dependence of the molecular oxygen admixture a different picture emerges. When the oxygen admixture is increased the plasma length decreases and the visible effluent intensity drops (see figures 6 and 7). In contrast to this declining behavior the ozone concentration increases. This converse characteristic can be explained by the manifold ways in which molecular species can get excited and form a significant energy or electron sink. In consequence the density of excited atomic argon species, which typically dominate the visible appearance of the visible effluent, rapidly decreases. Since a significant energy fraction is channeled into the molecular oxygen, formation of ozone as a reaction of atomic and molecular oxygen is enhanced. The results of our investigations with phase-resolved optical emission measurements and ozone absorption spectroscopy techniques distinctly show that the presence of reactive species is not necessarily connected to brightness and length of the jet's effluent. 


\subsection{Comparison of the ozone detection techniques}

In this study two different ozone detection techniques were employed: UV-absorption spectroscopy using radiation with a wavelength of about $254 \mathrm{~nm}$ and tuneable laser-absorption spectroscopy in the mid-IR spectral range at a wavenumber of $1027 \mathrm{~cm}^{-1}$. In both methods the ozone concentration is obtained by classical absorption technique and subsequent application of Beer's law. In contrast to the UV-absorption measurement, using the IR laser yields a significant higher spectral resolution. A better spectral resolution results in a more accurate measurement of the ozone concentration since a spectrally resolved absorption profile is yielded. This profile can be fitted with a theoretical profile to deduce the concentration. However, in the mid-IR region the absorption coefficient of ozone is much lower than in the UV-spectral range, which complicates absorption measurements on objects with short absorption length and small absorber concentration, as is the case in the jet's effluent. To bypass this disadvantage, the plasma jet was inserted inside a glass vessel and a multipass cell. The first leads to a higher amount of ozone in the direction of the laser beam and the latter significantly increases the absorption length. Together with the high spectral resolution discussed above a very accurate concentration measurement can be performed with this measurement technique. Even if no oxygen is admixed the ozone generation due to oxygen diffusing into the effluent from ambient air is detectable. In this case the measured ozone concentration amounts to $(7.7 \pm$ 1.1) $\times 10^{13} \mathrm{~cm}^{-3}$. This advantage in accuracy, however, goes along with a lack of spatial information, since the measured ozone concentration is the average concentration value inside the glass vessel. Nevertheless an ozone production rate $R_{\mathrm{O}_{3}}$ of the jet plasma can still be calculated taking into account the total gas flow rate $\Phi$ into the glass vessel. Along with the assumption that ozone is not chemically destroyed while it is in the vessel and that the measured average concentration $\left[\mathrm{O}_{3}\right]_{\mathrm{IR}}$ is equal to the concentration at the vessel outflow the ozone production rate in a stationary situation is

$$
R_{\mathrm{O}_{3}}=\left[\mathrm{O}_{3}\right]_{\mathrm{IR}} \cdot \Phi .
$$

The result of the ozone production rate determined from the IR-absorption measurement in dependence on the oxygen admixture is shown in figure 20. In contrast to the length of the effluent and the visible plasma intensity, the ozone production rate increases with increasing oxygen admixture. The highest value is found to be $2.3 \times 10^{17} \mathrm{~s}^{-1}$.

The ozone absorption measurement in the UV-spectral range yields space-resolved concentrations. This is the major advantage of this method compared with the used IRtechnique. Since no artificial absorption length enhancement was performed in the UV setup good accuracy was yielded by accumulating over a long time period. However, with the given setup, significant absorption can only be measured when oxygen is admixed to the jet. In order to quantitatively compare both techniques the production rate $R_{\mathrm{O}_{3}}$ is calculated also for the UV results: the measured ozone concentration $\left[\mathrm{O}_{3}\right]_{\mathrm{UV}}(r)$ at $z=4 \mathrm{~mm}$ is multiplied with the velocity profile $v(r)$ of the gas flow. This velocity profile at $z=4 \mathrm{~mm}$ was determined

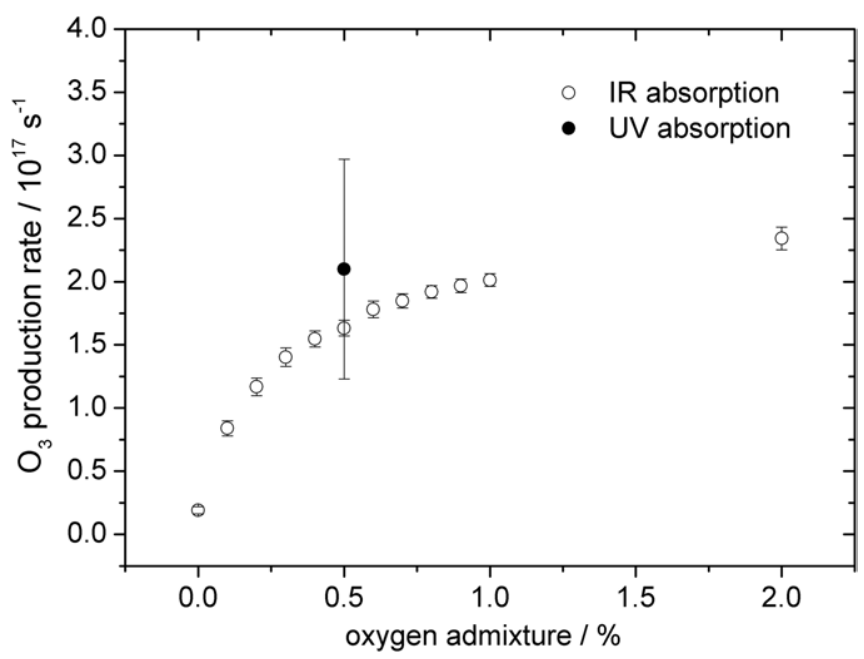

Figure 20. Ozone production rate of the investigated plasma jet in dependence on oxygen admixture determined with the IR-absorption technique (open circles) and UV-absorption technique (full circle).

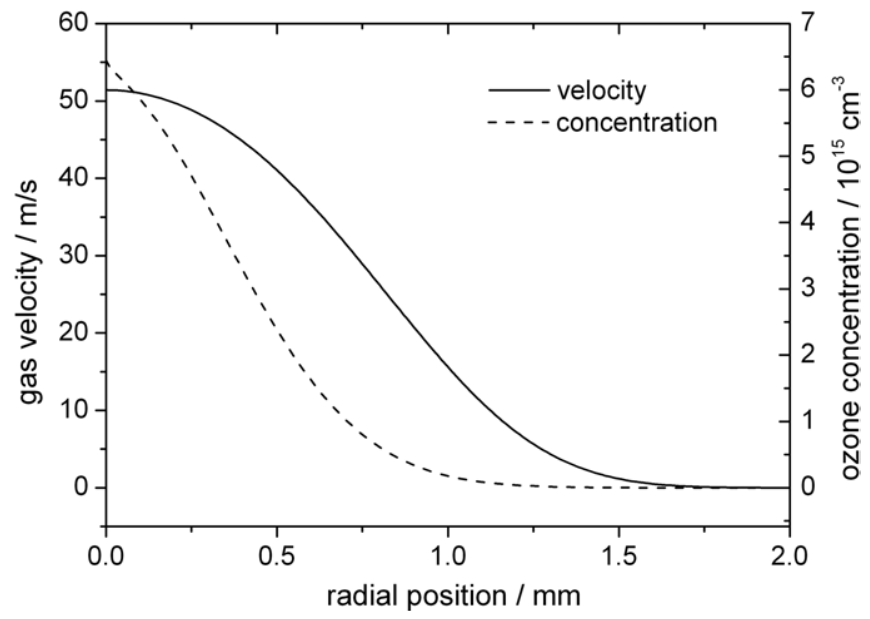

Figure 21. Radial gas velocity profile and ozone concentration profile at a nozzle distance of $z=4 \mathrm{~mm}$.

by solving the isothermal Navier-Stokes equation with the standard $k-\varepsilon$ turbulence model [24] using COMSOL 4.2 [25]. In order to obtain an accurate flow profile in the vicinity of the nozzle, the simulation domain contained the interior of the kINPen including the region around the pin-type electrode. As feed gas a $5 \mathrm{slm}$ argon flux with a density of $1.63 \mathrm{~kg} \mathrm{~m}^{-3}$ and a dynamic viscosity of $2.2 \times 10^{5} \mathrm{~kg} \mathrm{~m}^{-1} \mathrm{~s}^{-1}$ at room temperature is assumed. The result of this simulation is shown together with the ozone concentration profile $\left[\mathrm{O}_{3}\right]_{\mathrm{UV}}(r)$ in figure 21 . The concentration of ozone molecules in the radial direction corresponds to the velocity profile of the gas flow. Mostly only inside the gas flow channel of the jet are an appreciable number of ozone molecules present. This leads to the conclusion that at least for this short nozzle distance the ozone molecules are mainly created inside the plasma region and transported outward by the applied gas flow of the jet.

With both the velocity profile and concentration profile, the production rate

$$
R_{\mathrm{O}_{3}}=2 \pi \cdot \int_{0}^{\infty} v(r) \cdot\left[\mathrm{O}_{3}\right]_{\mathrm{UV}}(r) \cdot r \mathrm{~d} r
$$


is calculated. The result is shown as a full circle in figure 20 for the applied oxygen admixture of $0.5 \%$. Reasonably good agreement is found when comparing the value resulting from the UV-absorption measurement to that obtained from the IRabsorption technique. The ozone production rates, which are supposed to be equal for both experiments, differ only by a factor of 1.3. The reason for lower production rates in the IR-absorption measurement may be the destruction of ozone molecules at the vessel wall, which are not considered in the calculation of $R_{\mathrm{O}_{3}}$. This would be an additional loss term for ozone and in consequence lead to a lower measured production rate.

\section{Summary}

A cold atmospheric pressure argon plasma jet (kINPen) was investigated by optical and spectroscopic methods. Phaseresolved optical emission spectroscopy showed that the argon plasma jet is in fact a ( $1 \mathrm{MHz}$ ) bullet plasma jet. This is the first time that plasma bullets have been reported in a $\mathrm{MHz}$ argon atmospheric pressure plasma jet.

The time- and space-resolved measurements showed that the jet effluent length decreases with higher oxygen admixture, as can be expected. It is noted that the bullet velocity strongly depends on the oxygen admixture. The traveling velocity of the bullets in the order of $10^{4} \mathrm{~m} \mathrm{~s}^{-1}$ decreases with rising oxygen content in the feed gas.

The ozone density of the plasma jet was investigated by two complementary techniques: UV-absorption spectroscopy with high special resolution and mid-IR absorption spectroscopy with high sensitivity. Although the dynamic measurements show that the visible effluent length and intensity decrease, the obtained ozone production of the jet increases up to the maximum investigated molecular oxygen admixture of $2 \%$. A correlation of both complementary diagnostic techniques via the ozone production rate of the jet shows excellent agreement.

\section{Acknowledgments}

The authors thank Dr Andrei V Pipa for the discussions about IR spectroscopy and Dipl. Ing. Henrik Zimmermann for the technical support concerning the computational process and the Q-MACS implementation. The authors gratefully acknowledge the funding by the German Ministry of Education and Research (BMBF, grant number 03Z2DN12).

\section{References}

[1] Laroussi M 2009 IEEE Trans. Plasma Sci. 37 714-25

[2] Weltmann K D, Kindel E, Brandenburg R, Meyer C, Bussiahn R, Wilke C and von Woedtke T 2009 Contrib. Plasma Phys. 49 631-40

[3] Weltmann K-D, Kindel E, von Woedtke T, Hähnel M, Stieber M and Brandenburg R 2010 Pure Appl. Chem. 82 1223-37

[4] Reuter S, Niemi K, Schulz-von der Gathen V and Döbele H F 2009 Plasma Sources Sci. Technol. 18015006

[5] Reuter S, Winter J, Schmidt-Bleker A, Schroeder D, Lange H, Knake N, Schulz-von der Gathen V and Weltmann K-D 2012 Plasma Sources Sci. Technol. 21024005

[6] Pipa A V, Bindemann T, Foest R, Kindel E, Röpcke J and Weltmann K-D 2008 J. Phys. D: Appl. Phys. 41194011

[7] Weltmann K-D, Brandenburg R, von Woedtke T, Ehlbeck J, Foest R, Stieber M and Kindel E 2008 J. Phys. D: Appl. Phys. 41194008

[8] Niemi K, Reuter S, Graham L M, Waskoenig J, Knake N, Schulz-von der Gathen V and Gans T 2010 J. Phys. D: Appl. Phys. 43124006

[9] Gans T, Chun C Lin, Schulz-von der Gathen V and Doebele H F 2001 J. Phys. D: Appl. Phys. 34 L39-42

[10] Teschke M, Kedzierski J, Finantu-Dinu E G, Korzec D and Engemann J 2005 IEEE Trans. Plasma Sci. 33 310-1

[11] Lu X P and Laroussi M 2006 J. Appl. Phys. 100063302

[12] Bussiahn R, Kindel E, Lange H and Weltmann K-D 2010 J. Phys. D: Appl. Phys. 43165201

[13] Topala I and Dumitrascu N 2011 IEEE Trans. Plasma Sci. 39 2342-3

[14] Kim D B, Jung H, Gweon B and Choe W 2010 Phys. Plasmas 17073503

[15] Sands B L, Ganguly B N and Tachibana K 2008 Appl. Phys. Lett. 92151503

[16] Karakas E and Laroussi M 2010 J. Appl. Phys. 108063305

[17] Hoder T, Brandenburg R, Basner R, Weltmann K-D, Kozlov K V and Wagner H-E 2010 J. Phys. D: Appl. Phys. 43124009

[18] Gerling T, Nastuta A V, Bussiahn R, Kindel E and Weltmann K-D 2012 Plasma Sources Sci. Technol. 21034012

[19] Orphal J 2003 J. Photochem. Photobiol. A 157 185-209

[20] Q-MACSoft-HT online available: http://www.neoplas-control.de/

[21] Rothman L S et al 2005 J. Quant. Spectrosc. Radiat. Transf. 96 139-204

[22] Stancu G D 2004 PhD Thesis Ernst-Moritz-Arndt University, Greifswald

[23] Pipa A V and Röpke J 2009 IEEE Trans. Plasma Sci. 37 1000-3

[24] Wilcox D C 1998 Turbulence Modeling for CFD 2nd edn (DCW Industries, Canada)

[25] 2011 COMSOL 4.2 (Burlington, MA: COMSOL) 